Georgian Mathematical Journal

Volume 12 (2005), Number 2, 283-307

\title{
BOUNDARY REGULARITY FOR CAPILLARY SURFACES
}

\author{
FEI-TSEN LIANG
}

\begin{abstract}
For solutions of capillarity problems with the boundary contact angle being bounded away from 0 and $\pi$ and the mean curvature being bounded from above and below, we show the Lipschitz continuity of a solution up to the boundary locally in any neighborhood in which the solution is bounded and $\partial \Omega$ is $C^{2}$; the Lipschitz norm is determined completely by the upper bound of $|\cos \theta|$, together with the lower and upper bounds of $H$, the upper bound of the absolute value of the principal curvatures of $\partial \Omega$ and the dimension $n$.
\end{abstract}

2000 Mathematics Subject Classification: 35J60, 53A10.

Key words and phrases: Capillary surface, boundary regularity.

\section{INTRODUCTION}

Given a domain $\Omega \subset \mathbb{R}^{n}, n \geq 2$, let $H(x, u(x))$ be a given Lipschitz continuous function in $\Omega \times \mathbb{R}$. A solution of the capillarity problem can be regarded at as a solution of the equation of surfaces of the prescribed mean curvature

$$
\operatorname{div} T u=H(x, u) \text { in } \Omega,
$$

subject to the "contact angle" boundary condition

$$
T u \cdot \nu=\cos \theta,
$$

where

$$
T u=\frac{D u}{\sqrt{1+|D u|^{2}}},
$$

$D u=\left(\partial u / \partial x_{1}, \partial u / \partial x_{2}, \ldots, \partial u / \partial x_{n}\right)$ and $\nu$ is the outward pointing unit normal to $\partial \Omega$. Thus, geometrically, we are looking for a function $u$ over $\bar{\Omega}$ whose graph has the prescribed mean curvature $H$ and which meets the boundary cylinder in the prescribed angle $\theta . H=H(x, t)$ is assumed to be a given locally Lipschitz function on $\bar{\Omega} \times \mathbb{R}$ satisfying the structural condition

$$
\frac{\partial H}{\partial t}(x, t) \geq 0, \quad \text { for } x \in \Omega, \quad t \in \mathbb{R} .
$$

As (0.1) is the Euler equation of the functional

$$
I(v)=\int_{\Omega} \sqrt{1+|D v|^{2}} d x+\int_{\Omega} \int_{0}^{v} H(x, t) d t d x,
$$


it corresponds to the following variational problem for the capillarity problem:

$$
I(v)+\int_{\partial \Omega} \cos \theta v d \mathcal{H}_{n-1} \rightarrow \min , \text { for all } v \in B V(\Omega),
$$

with $\mathcal{H}_{n-1}$ being the $(n-1)$-dimensional Hausdorff measure.

We are interested in regularity near the boundary $\partial \Omega$ for solutions $u \in C^{2}(\Omega)$. In this work our main interest is in the case where $|\cos \theta|$ is bounded away from 0 and 1 and the mean curvature $H$ is bounded from above and below. We shall show that in such a case the solution is Lipschitz continuous up to the boundary locally in any neighborhood in which $u$ is bounded and $\partial \Omega$ is $C^{2}$; the Lipschitz norm is determined completely by the upper and lower bounds of $|\cos \theta|$, together with the lower and upper bounds of $H$, the upper bound of the absolute value of the principal curvatures of $\partial \Omega$ and $n$.

Spruck and Simon treat in [12] the case where $\Omega$ is $C^{4}, \theta$ in $(0.2)$ is $C^{1, \alpha}$ on $\partial \Omega$ for some $0<\alpha<1$, and $H(x, t)$ is strictly monotone in $t$ :

$$
\inf _{x \in \bar{\Omega} ; t \in \mathbb{R}} \frac{\partial H}{\partial t}(x, t)>0 .
$$

In case $0<\theta<\pi$, the existence of a $C^{2}(\bar{\Omega})$ solution of $(0.1)$ and $(0.2)$ is established in [12]. In case $\theta$ is allowed to take 0 and/or $\pi$, setting

$$
\begin{aligned}
S_{1}^{+} & =\{x: x \in \partial \Omega, \theta \equiv 0 \text { in some neighborhood of } x\} \\
S_{1}^{-} & =\{x: x \in \partial \Omega, \theta \equiv \pi \text { in some neighborhood of } x\} \\
S_{2} & =\{x: x \in \partial \Omega, 0<\theta<\pi\}
\end{aligned}
$$

a function $u \in C^{2}\left(\Omega \cup S_{2}\right)$ is shown to exist in [12], which satisfies (0.1) in $\Omega$ and satisfies (0.2) on $S_{2}$; furthermore, $u$ is Hölder continuous at each point of $S_{1}^{+} \cup S_{1}^{-}$, has a restriction to $\partial \Omega$ which is Lipschitz continuous at each point of $S_{1}^{+} \cup S_{1}^{-}$in the sense that

$$
\lim _{\varepsilon^{+} \rightarrow 0} \int_{U \cap \Omega_{\varepsilon}}|T u \cdot \nu \pm 1| d x=0 \text { for each } U \subset \Omega \text { with } \bar{U} \cap \partial \Omega \subset S_{1}^{\mp},
$$

assuming that $T u$ is extended to some boundary strip $\Omega_{\varepsilon}$ with width $\varepsilon$ so that it is constant along the normals to $\partial \Omega$. This result is obtained first by establishing estimates of tangential derivatives under the condition that $|\cos \theta| \leq \gamma<1$ for some positive constant $\gamma$; in case $\theta$ is constant in a neighborhood of the point under consideration, the estimates of tangential derivatives are independent of $\gamma$. This proves the Lipschitz continuity of the trace of $u$ on $\partial \Omega$, which and the result in [10] yield the Hölder continuity of $u$. Estimates for the tangential derivatives are obtained by performing the transformation of the coordinates near the boundary analogously to that in [11], with a subsequent differentiation of (0.1), (0.2) and substituting (0.6) into the resultant identities. The disadvantage of their proofs is that $H$ has to be assumed to satisfy the strict inequality (0.6) rather than the less restrictive condition (0.4). 
In contrast, the following estimates for the boundary oscillation of $u$ are established in [8, Main Theorem III].

Theorem 1. Let $u \in C(\bar{\Omega})$ be a bounded solution of (0.1) and (0.2) in $\Omega$ in the sense that

$$
\begin{aligned}
& \int_{\Omega} \frac{D u}{\sqrt{1+|D u|^{2}}} \cdot D \eta d x+\int_{\Omega} H \cdot \eta d x \\
&-\int_{\partial \Omega} \beta \cdot \eta d \mathcal{H}_{n-1}=0 \text { for all } \eta \in H^{1,1}(\Omega),
\end{aligned}
$$

and with $\beta=\cos \theta$. Suppose that for two positive constant $\tilde{\tilde{\beta}}, \tilde{\beta} \leq 1$ and a ball $B_{R}\left(x_{0}\right)$ intersecting the interior of $\Omega$, the function $\cos \theta$ is continuous on $\partial \Omega \cap B_{R}\left(x_{0}\right)$ and there holds

$$
0<\tilde{\tilde{\beta}} \leq|\cos \theta| \leq \tilde{\beta}<1
$$

for all $x \in \partial \Omega \cap B_{R}\left(x_{0}\right)$, and such that

$$
\hat{H}^{ \pm}(x)=H\left(x, \pm \inf _{\partial \Omega} u\right) \in L^{p}(\Omega), \quad \hat{\hat{H}}^{ \pm}(x)=H\left(x, \pm \sup _{\partial \Omega} u\right) \in L^{p}(\Omega)
$$

and

$$
H(x, 0) \in L^{1}(\Omega) .
$$

Suppose $\partial \Omega$ is piecewise Lipschitz continuous with possible outward and/or inward cusps. Then the trace of $u$ on $\partial \Omega$ is Lipschitz continuous locally in $\partial \Omega \cap B_{R}\left(x_{0}\right)$ if $\partial \Omega \cap B_{R}\left(x_{0}\right)$ is either $C^{2}$ or is the graph of a Lipschitz continuous function with Lipschitz constant $L$ such that $\tilde{\beta} \sqrt{1+L^{2}}<1$. The Lipschitz constant $L_{0}(\tilde{\beta}, \tilde{\tilde{\beta}})$ of the trace of $u$ on $\partial \Omega \cap B_{R}\left(x_{0}\right)$ depends only on $H, n$, together with the constants $\tilde{\beta}$, $\tilde{\tilde{\beta}}$ and $\tilde{\mathcal{K}}_{\partial \Omega \cap B_{R}\left(x_{0}\right)}$, where for a set $A$, we set $\mathcal{K}_{\partial \Omega \cap A}=\mathcal{K}_{\partial \Omega \cap A}$ in case $\partial \Omega \cap A$ is $C^{2}$ and $\mathcal{K}_{\partial \Omega \cap A}=\sqrt{1+L^{2}}$ in case $\partial \Omega \cap A$ is Lipschitz continuous with Lipschitz constant L; here $\mathcal{K}_{\partial \Omega \cap A}$ is an upper bound for the absolute value of the principal curvatures of $\partial \Omega \cap A$ in case $\partial \Omega \cap A$ is $C^{2}$.

We notice that (0.9) and (0.10) hold in particular if $|H(x, t)|$ is bounded in $\bar{\Omega} \times \mathbb{R}$.

The following global estimates for $u$ are also established in [8, Main Theorem IV].

Theorem 2. Suppose that $\partial \Omega$ is Lipschitz continuous without outward cusps. Suppose that (0.10) holds and

$$
H_{t_{0}} \in L^{p}(\Omega), \quad \text { for some } p>n \text { and } t_{0} \in \mathbb{R} \text {. }
$$

If $u \in C(\bar{\Omega})$ is a solution to (0.1) and (0.2) in $\Omega$ such that (0.2) is fulfilled in the sense of $(0.7)$ and if $\cos \theta(x)=\beta(x)$ satisfies the condition (0.8) for all $x \in \partial \Omega$ 
and is piecewise continuous on $\partial \Omega$, then

$$
\sup _{\Omega} u-\inf _{\Omega} u
$$

can be estimated in terms of $t_{0}, n,\left\|H_{t_{0}}\right\|_{L^{p}(\Omega)}, \int_{\Omega} H(x, 0) d x$, the constant $\tilde{\beta}$ and the constant $\tilde{\mathcal{K}_{\partial \Omega}}$ which depends only on the geometry of $\Omega$; here $\tilde{\mathcal{K}_{\partial \Omega}}=$ $\max _{i \in \mathbb{I}} \mathcal{K}_{\partial \tilde{\Omega} \cap A_{i}}$, in which $\left\{\Omega \cap A_{i}\right\}_{i \in \mathbb{I}}$ is a covering of $\partial \Omega$ such that $\partial \Omega \cap A_{i}, i \in \mathbb{I}$, is either $C^{2}$ or is a Lipschitz function with Lipschitz constant $L, \tilde{\beta} \cdot \sqrt{1+L^{2}}<1$.

The results in the previous two theorems are established by modifying the approach taken in [2], [3] and [4], which is based on the minimizing property (0.5) $u$ satisfies and the iteration technique used in [13].

Below we give the result, in which we let the set $A_{r}(\hat{x})$, for some small positive number $r$, be chosen as follows. Namely, setting

$$
\partial^{*} \Omega_{t}=\{x: x \in \Omega, \operatorname{dist}(x, \partial \Omega)=t\} \text { for } t>0,
$$

we let the boundary $\partial\left(\Omega \cap A_{r}(\hat{x})\right)$ be made up of three parts, namely

$$
\partial\left(\Omega \cap A_{r}(\hat{x})\right)=\left(\partial \Omega \cap \overline{A_{r}(\hat{x})}\right) \cup\left(\partial^{*} A_{r}(\hat{x})\right) \cup\left(\partial^{* *} A_{r}(\hat{x})\right),
$$

such that

$$
\begin{aligned}
\partial^{*} A_{r}(\hat{x}) & =\partial A_{r}(\hat{x}) \cap \partial^{*} \Omega_{r}, \\
\partial^{* *} A_{r}(\hat{x}) & =\left(\partial A_{r}(\hat{x}) \cap \Omega\right) \backslash \partial^{*} \Omega_{r},
\end{aligned}
$$

and

$$
\left.D d \cdot \nu_{\Omega \cap A_{r}(\hat{x})}\right|_{\partial^{* *} A_{r}(\hat{x})}=0,
$$

where we let $\nu_{\Omega \cap A_{r}(\hat{x})}$ be the unit outward normal to $\partial\left(\Omega \cap A_{r}(\hat{x})\right)$; furthermore,

$$
\begin{gathered}
\operatorname{diam}\left(\partial \Omega \cap \overline{A_{r}(\hat{x})}\right) \leq r \quad \text { and } \quad \operatorname{diam}\left(\partial^{*} A_{r}(\hat{x})\right) \leq r \\
\left|\partial \Omega \cap \overline{A_{r}(\hat{x})}\right| \geq\left(\frac{r}{2}\right)^{n-1} \quad \text { and } \quad\left|\partial^{*} A_{r}(\hat{x})\right| \geq\left(\frac{r}{2}\right)^{n-1} .
\end{gathered}
$$

Proposition 1. Let $u \in C(\bar{\Omega})$ be a solution to $(0.1)$ and $(0.2)$ in $\Omega$ such that $(0.2)$ be fulfilled in the sense of (0.7). Suppose that for a constant $H_{*}$,

$$
|H(x, t)| \leq H_{*} \text { for }(x, t) \in \bar{\Omega} \times \mathbb{R} .
$$

Suppose that for a positive constant $\tilde{\beta}<1$ and a point $x_{0} \in \partial \Omega$, the function $\cos \theta$ is continuous in $\partial \Omega \cap A_{\delta_{0}}\left(x_{0}\right)$ and there holds

$$
0<\tilde{\tilde{\beta}}<\cos \theta \leq \tilde{\beta} \quad \text { or } \quad 0>-\tilde{\tilde{\beta}}>\cos \theta>-\tilde{\beta} \quad \text { for } \quad x \in \partial \Omega \cap \overline{A_{\delta_{0}}\left(x_{0}\right)} \text {. }
$$

Suppose that $\partial \Omega \cap \overline{A_{\delta_{0}}\left(x_{0}\right)}$ is of the class $C^{2}$ and

$$
\lim _{x_{k} \rightarrow x_{0}}|D u| \geq \frac{2}{\tilde{\beta}}
$$


for each subsequence of points $x_{k}$ approaching $x_{0}$. Then, setting $R=\left(\delta_{0} / 4\right)^{1+\varepsilon_{0}}$, $\varepsilon_{0}$ being a positive number which can be arbitrarily small, there hold, for $\delta_{0}$ sufficiently small, respectively,

$$
\begin{aligned}
u(x)-\inf _{\Omega \cap A_{R}\left(x_{0}\right)} u \leq & \mathfrak{C} \cdot \hat{\mathfrak{C}} \cdot\left(\delta_{0}\right)^{1-n \varepsilon_{0}}+2\left(\delta_{0}\right)^{1+\varepsilon_{0}} \\
& +\mathfrak{C} \cdot \hat{\mathfrak{C}} \cdot\left(\delta_{0}\right)^{1-n \varepsilon_{0}} \cdot\left(\sup _{\Omega \cap A_{\delta_{0}}\left(x_{0}\right)} u-\inf _{\Omega \cap A_{\delta_{0}}\left(x_{0}\right)} u\right)
\end{aligned}
$$

if the first case in (0.12) holds, and

$$
\begin{aligned}
\sup _{\Omega \cap A_{R}\left(x_{0}\right)} u-u(x) \leq & \mathfrak{C} \cdot \hat{\mathfrak{C}} \cdot\left(\delta_{0}\right)^{1-n \varepsilon_{0}}+2\left(\delta_{0}\right)^{1+\varepsilon_{0}} \\
& +\mathfrak{C} \cdot \hat{\mathfrak{C}} \cdot\left(\delta_{0}\right)^{1-n \varepsilon_{0}} \cdot\left(\sup _{\Omega \cap A_{\delta_{0}}\left(x_{0}\right)} u-\inf _{\Omega \cap A_{\delta_{0}}\left(x_{0}\right)} u\right), \\
\mathfrak{C}= & 2^{n+7} \cdot(n+1) \cdot k_{(n+1)} \cdot[1+(3 / \hat{\beta})],
\end{aligned}
$$

if the second case in $(0.12)$ holds, where $k_{(n+1)}$ is the isoperimetric constant in $\mathbb{R}^{n+1}$ and $\hat{\mathfrak{C}}$ is a constant determined by $\tilde{\beta}, \tilde{\tilde{\beta}}, H_{*}, \mathcal{K}_{\partial \Omega \cap \overline{A_{\delta_{0}}\left(x_{0}\right)}}$ and $n$.

From the interior regularity of $u$ and (0.12), we obtain in Appendix 3

$$
\begin{array}{r}
\tilde{\tilde{\beta}} / 2<\cos \theta \leq(1+\tilde{\beta}) / 2 \quad \text { or } \quad-\tilde{\tilde{\beta}} / 2>\cos \theta>-(1+\tilde{\beta}) / 2 \\
\text { for } x \in \partial^{*} A_{\delta_{0}}\left(x_{0}\right) .
\end{array}
$$

Using this and the interior regularity of $u$, we obtain

Theorem 3. Let $u \in C(\bar{\Omega})$ be a solution to $(0.1)$ and $(0.2)$ in $\Omega$ such that $(0.2)$ be fulfilled in the sense of $(0.7)$ and such that $H$ and $\cos \theta$ satisfy respectively (0.11) and (0.12). Suppose that $\partial \Omega \cap \overline{A_{\delta_{0}}\left(x_{0}\right)}$ is of the class $C^{2}$. If

$$
|D u(x)| \geq \frac{5}{\tilde{\beta}}, \quad \text { for a point } x \in \partial^{*} A_{\delta_{0}}\left(x_{0}\right)
$$

then, for $x \in \partial^{*} A$, there holds

$$
\begin{aligned}
u(x)-\inf _{\Omega \cap A_{R}\left(x_{0}\right)} u \leq & \mathfrak{C} \cdot \hat{\mathfrak{C}}_{*}^{*} \cdot\left(\delta_{0}\right)^{1-n \varepsilon_{0}}+2\left(\delta_{0}\right)^{1+\varepsilon_{0}} \\
& +\mathfrak{C} \cdot \hat{\mathfrak{C}}_{*}^{*} \cdot\left(\delta_{0}\right)^{1-n \varepsilon_{0}} \cdot\left(\sup _{\Omega \cap A_{\delta_{0}}\left(x_{0}\right)} u-\inf _{\Omega \cap A_{\delta_{0}}\left(x_{0}\right)} u\right)
\end{aligned}
$$

if the first case in (0.12) holds, and

$$
\begin{aligned}
\sup _{\Omega \cap A_{R}\left(x_{0}\right)} u-u(x) \leq & \mathfrak{C} \cdot \hat{\mathfrak{C}}_{*} \cdot\left(\delta_{0}\right)^{1-n \varepsilon_{0}}+2\left(\delta_{0}\right)^{1+\varepsilon_{0}} \\
& +\mathfrak{C} \cdot \hat{\mathfrak{C}}_{*} \cdot\left(\delta_{0}\right)^{1-n \varepsilon_{0}} \cdot\left(\sup _{\Omega \cap A_{\delta_{0}}\left(x_{0}\right)} u-\inf _{\Omega \cap A_{\delta_{0}}\left(x_{0}\right)} u\right)
\end{aligned}
$$

if the second case in (0.12) holds, where $\hat{\mathfrak{C}}_{*}$ is a constant determined by $\tilde{\beta}, \tilde{\tilde{\beta}}$, $H_{*}, \mathcal{K}_{\partial \Omega \cap \overline{A_{\delta_{0}}\left(x_{0}\right)}}$ and $n$.

Letting $\varepsilon_{0} \rightarrow 0$ in Theorem 3 and combining the latter theorem with Theorem 2 , we obtain 
Main Theorem. Let $u \in C(\bar{\Omega})$ be a solution to (0.1) and (0.2) in $\Omega$ such that (0.2) is fulfilled in the sense of (0.7). Let $|H(x, t)|$ be bounded by the constant $H_{*}$ in $\bar{\Omega} \times \mathbb{R}$. Suppose that for a positive constant $\tilde{\beta}<1$, the inequalities $(0.12)$ hold for $x_{0}$ and $A_{\delta_{0}}\left(x_{0}\right), \delta_{0}$ being sufficiently small and $x_{0} \in \partial \Omega$. Suppose that $\delta_{0}$ is so small that $\partial \Omega \cap \overline{A_{\delta_{0}}\left(x_{0}\right)}$ is of class $C^{2}$. Then $u$ is Lipschitz continuous in $\Omega \cap A_{\delta_{0}}\left(x_{0}\right)$ up to the boundary; the Lipschitz norm of $u$ in $\overline{\Omega \cap A_{\delta_{0}}\left(x_{0}\right)}$ is either less than $\frac{5}{\tilde{\beta}}$ or is determined by $H_{*}, n, \tilde{\beta}, \tilde{\tilde{\beta}}, \mathcal{K}_{\partial \Omega \cap \overline{A_{r}(\hat{x})}}$, and $|\Omega|$.

Here and in the following, we denote by $|\cdot|$ either an $n$-dimensional or an $(n+1)$-dimensional Hausdorff measure.

Proposition 1 is based on the reasoning in Giusti [6, pp. 312-313] which leads to estimates for the oscillation of $u$ in terms of the $L^{1}$-norm of $u$, under the conditions (1.1) or (1.3) indicated below, which says that the subgraph of $u$ or the complement of the subgraph of $u$ includes a large portion of a sufficiently small cylinder-type region around $u\left(x_{0}\right), x_{0} \in \partial \Omega$. This reasoning is given in Subsection 1. In Subsection 2, we formulate a result which is essentially Theorem 3.2 in Giusti [6] and which assures us of the fulfillment of (1.1) or (1.3) for capillarity surfaces. This suggests us that we should estimate the $L^{1}$ norm of $u$ by writing (0.1) and (0.2) in weak a form in which the assumed boundedness of $|u|$ allows us to take the test function to be $\left(u(x)-\inf _{\Omega} u\right)$ or $(\sup u-u(x))$. The resultant inequalities (1.7) and (1.8) suggest us to restrict our consideration to a small region $\Omega \cap A$ of the type indicated in the beginning of Subsection 4 which is analogous to that of $A_{R}\left(x_{0}\right)$, for which the resultant boundary integrals are treated in Subsection 3.2. To proceed with obtaining $L^{1}$ estimates of $\left(u(x)-\inf _{\Omega \cap A} u\right)$ and $\left(\sup _{\Omega \cap A} u-u(x)\right)$ in $\Omega \cap A$, we shall appeal to the modified Sobolev inequality given in Proposition A.1 in Appendix I, for which we have to estimate $\int_{\partial^{* *} A}\left(\sup _{\Omega \cap A} u-u(x)\right) d \mathcal{H}_{n-1}$ and $\int_{\partial^{* *} A}\left(u(x)-\inf _{\Omega \cap A} u\right) d \mathcal{H}_{n-1}$ with an application of the condition (0.11).

\section{Proof of Proposition 1}

1. Oscillation of $\mathbf{u}$ in terms of the $\mathbf{L}^{1}$-norm of $u$. We modify the approach taken by Giusti [6, pp. 312-313].

Let $u$ be a function with the subgraph

$$
U=\{(x, t) \in \Omega \times \mathbb{R}, t<u(x)\},
$$

and set for points $\hat{z}=(\hat{x}, \hat{t}) \in \Omega \times \mathbb{R}$ and for $r>0$,

$$
U_{r}(\hat{z})=C_{r}(\hat{z}) \cap U \quad \text { and } \quad U_{r}^{\prime}(\hat{z})=C_{r}(\hat{z}) \backslash U,
$$

where

$$
C_{r}(\hat{z})=\left\{(x, t): x \in A_{r}(\hat{x}),|t-\hat{t}|<r\right\},
$$

with $A_{r}(\hat{x})$ being chosen as indicated before Theorem 3 . 
We make the assumption that there exist positive constants $R_{-}$and $\alpha_{*}$ such that

$$
\begin{aligned}
& \left|U_{r}(\hat{z})\right|>\alpha_{*} r^{n+1} \quad \text { for every } r \leq R_{-}, \\
& \quad \text { whenever }\left|U_{r}(\hat{z})\right|>0 \text { for every } r>0 .
\end{aligned}
$$

Suppose $x_{0} \in \mathbb{R}^{n}$ such that $A_{R}\left(x_{0}\right) \cap \Omega$ is nonempty. Let us set

$$
M_{R}=\sup _{\Omega \cap A_{R}\left(x_{0}\right)} u, \text { and } m_{R}=\inf _{\Omega \cap A_{R}\left(x_{0}\right)} u .
$$

We shall establish below the fulfilment of the following inequality under the assumption that (1.1) holds; namely, for $R \leq R_{-}$:

$$
u\left(x_{0}\right)-m_{R} \leq \frac{2^{n+2}}{\alpha_{*} R^{n}} \int_{\Omega \cap A_{R}\left(x_{0}\right)}\left(u(x)-m_{R}\right) d x+2 R .
$$

Indeed, let

$$
z_{j}=\left(x_{0}, m_{R}+2 j R\right), \text { for } j \in \mathbb{N} \text {. }
$$

Then

$$
z_{j} \in U, \quad \text { for } j \leq j_{*}=\left[\frac{u\left(x_{0}\right)-m_{R}}{2 R}\right],
$$

where $[s]$ denotes the largest integer less than $s$ for $s>0$. Under the assumption (1.1), we have

$$
\left|U_{R / 2}\left(z_{j}\right)\right| \geq \alpha_{*}\left(\frac{R}{2}\right)^{n+1}, \quad \text { for } 1 \leq j \leq j_{*},
$$

and therefore

$$
\int_{\Omega \cap A_{R}\left(x_{0}\right)}\left(u(x)-m_{R}\right) d x \geq \sum_{j=1}^{j_{*}}\left|U_{R / 2}\left(z_{j}\right)\right| \geq j_{*} \cdot \alpha_{*} \cdot\left(\frac{R}{2}\right)^{n+1} .
$$

Hence

$$
\begin{aligned}
M_{R} & =u\left(x_{0}\right)+\left(M_{R}-u\left(x_{0}\right)\right) \leq 2\left(j_{*}+1\right) R+m_{R}+\left(M_{R}-u\left(x_{0}\right)\right) \\
& \leq \frac{2^{n+2}}{\alpha_{*} R^{n}} \int_{\Omega \cap A_{R}\left(x_{0}\right)}\left(u(x)-m_{R}\right) d x+2 R+m_{R}+\left(M_{R}-u\left(x_{0}\right)\right),
\end{aligned}
$$

which is $(1.2)$.

Assume now, instead of (1.1), that there exist positive constants $R_{+}$and $\alpha_{*}$ such that

$$
\begin{aligned}
& \left|U_{r}^{\prime}(\hat{z})\right|>\alpha_{*} r^{n+1} \quad \text { for every } \quad r \leq R_{+} \\
& \quad \text { whenever }\left|U_{r}^{\prime}(\hat{z})\right|>0 \text { for every } r>0 .
\end{aligned}
$$


Under the assumption (1.3), we shall analogously obtain, for $R \leq R_{+}$, the inequality

$$
M_{R}-u\left(x_{0}\right) \leq \frac{2^{n+2}}{\alpha_{*} R^{n}} \int_{\Omega \cap A_{R}\left(x_{0}\right)}\left(M_{R}-u(x)\right) d x+2 R .
$$

Indeed, let

$$
z_{j}^{+}=\left(x_{0}, M_{R}-2 j R\right), \text { for } j \in \mathbb{N}
$$

Then

$$
z_{j}^{+} \in U^{\prime}=(\Omega \times \mathbb{R}) \backslash U, \quad \text { for } j \leq j_{*}^{+}=\left[\frac{M_{R}-u\left(x_{0}\right)}{2 R}\right] .
$$

By assumption, we have

$$
\left|U_{R / 2}\left(z_{j}^{+}\right)\right| \geq \alpha_{*}\left(\frac{R}{2}\right)^{n+1}, \quad \text { for } 1 \leq j \leq j_{*}^{+},
$$

and therefore

$$
\int_{\Omega \cap A_{R}\left(x_{0}\right)}\left(M_{R}-u\left(x_{0}\right)\right) d x \geq \sum_{j=1}^{j_{*}}\left|U_{R / 2}^{\prime}\left(z_{j}^{+}\right)\right| \geq j_{*}^{+} \cdot \alpha_{*} \cdot\left(\frac{R}{2}\right)^{n+1},
$$

which yields

$$
\begin{aligned}
-m_{R} & =u\left(x_{0}\right)+\left(u\left(x_{0}\right)-m_{R}\right) \leq 2\left(j_{*}^{+}+1\right) R-M_{R}+\left(u\left(x_{0}\right)-m_{R}\right) \\
& \leq \frac{2^{n+2}}{\alpha_{*} R^{n}} \int_{\Omega \cap A_{R}\left(x_{0}\right)}\left(M_{R}-u(x)\right) d x+2 R-M_{R}+\left(u\left(x_{0}\right)-m_{R}\right) ;
\end{aligned}
$$

this is (1.4).

2. (1.1) or (1.3) for capillary surfaces. The above consideration suggests that we should apply the estimates in Giusti [6, Theorem 3.2]. Indeed, below we appeal to estimates in Proposition 1, which are essentially obtained in Giusti [6, Theorem 3.2] and which can be proved by the argument given in [7, Appendix] without any essential modification.

Proposition 2. Let $u$ be a solution to (0.5) with subgraph U. Suppose that $\partial \Omega \cap \overline{A_{R_{0}}\left(x_{0}\right)}$ is of the class $C^{2}$ whose principal curvatures are bounded in the absolute value by $\mathcal{K}_{\partial \Omega \cap \overline{A_{R_{0}}\left(x_{0}\right)}}$. If there exists a constant $\hat{\gamma}, 0 \leq \hat{\gamma}<1$, such that

$$
\beta(x) \geq-\hat{\gamma}, \text { for all } x \in \partial \Omega \cap \overline{A_{R_{0}}\left(x_{0}\right)},
$$

and if

$$
\left|U_{r}(\hat{z})\right|>0, \text { for all } r>0
$$

then there exist positive constants $R_{-}$and $\alpha_{*}$ determined completely by $n$, $\inf _{\Omega \times \mathbb{R}} H, \hat{\gamma}, \mathcal{K}_{\partial \Omega \cap \overline{A_{R_{0}}\left(x_{0}\right)}}, R_{0}$ and the largest possible radius $R_{\Omega}$ of the inscribed disks in $\Omega$ such that

$$
\left|U_{r}(\hat{z})\right|>\alpha_{*} r^{n+1}, \text { for every } r \leq R_{-} .
$$


In particular, we can take

$$
\alpha_{*}=\frac{1-\hat{\gamma}}{16(n+1) k_{(n+1)}},
$$

with $k_{(n+1)}$ being the isoperimetric constant in $\mathbb{R}^{n+1}$, and

$$
R_{-}= \begin{cases}\min \left(\frac{C_{\hat{\gamma}}}{C_{\varepsilon, \Omega} k_{(n+1)}}, R_{0}\right), & \text { if } \inf _{\Omega \times \mathbb{R}} H \geq 0, \\ \min \left(\frac{C_{\hat{\gamma}}^{-}}{2^{1 /(n+1)} \cdot C_{\varepsilon, \Omega} \cdot k_{(n+1)}}, \tilde{R}_{-}\right), R_{0}, & \text { if } \inf _{\Omega \times \mathbb{R}} H<0,\end{cases}
$$

in which we set

$$
\begin{gathered}
C_{\hat{\gamma}}=\min \left(\frac{1}{2}, \frac{1-\hat{\gamma}}{3 \hat{\gamma}+1}\right) \\
C_{\varepsilon, \Omega}=\frac{2}{\varepsilon}+2(n-1) \mathcal{K}_{\partial \Omega}, \quad \text { with } \varepsilon \leq \min \left(\frac{1}{2 \mathcal{K}_{\partial \Omega}}, R_{\Omega}\right) \\
\tilde{R}_{-}=\left(\frac{1-\hat{\gamma}}{4 n k_{(n+1)} \cdot\left|\inf _{\Omega \times \mathbb{R}} H\right|}\right)^{n+1}
\end{gathered}
$$

and

$$
C_{\hat{\gamma}}^{-}=\min \left(\frac{1}{2}, \frac{1-\hat{\gamma}-2 n k_{(n+1)} \cdot\left|\inf _{\Omega \times \mathbb{R}} H\right| \cdot\left(\tilde{R}_{-}\right)^{n}}{3 \hat{\gamma}+1}\right) .
$$

If there exists a constant $\hat{\gamma}, 0 \leq \hat{\gamma}<1$, such that

$$
\beta(x) \leq \hat{\gamma}, \text { for all } x \in \partial \Omega \cap \overline{A_{R_{0}}\left(x_{0}\right)},
$$

and if

$$
\left|U_{r}^{\prime}(\hat{z})\right|>0, \text { for all } r>0
$$

then there exists a positive constant $R_{+}$determined completely by $n, \sup _{\Omega \times \mathbb{R}} H, \hat{\gamma}$, $\mathcal{K}_{\partial \Omega \cap \overline{A_{R_{0}}\left(x_{0}\right)}}, R_{0}$ and $R_{\Omega}$ such that

$$
\left|U_{r}^{\prime}(\hat{z})\right|>\alpha_{*} r^{n+1}, \text { for every } r \leq R_{+},
$$

for the same constant $\alpha_{*}$ as above. In particular, we can take

$$
R_{+}= \begin{cases}\min \left(\frac{C_{\hat{\gamma}}}{C_{\varepsilon, \Omega} k_{(n+1)}}, R_{0}\right), & \text { if } \sup _{\Omega \times \mathbb{R}} H \leq 0, \\ \min \left(\frac{C_{\hat{\gamma}}^{+}}{2^{1 /(n+1)} \cdot C_{\varepsilon, \Omega} \cdot k_{(n+1)}}, \tilde{R}_{+}, R_{0}\right), & \text { if } \sup _{\Omega \times \mathbb{R}} H>0,\end{cases}
$$

with

$$
\tilde{R}_{+}=\left(\frac{1-\hat{\gamma}}{4 n k_{(n+1)} \cdot\left|\sup _{\Omega \times \mathbb{R}} H\right|}\right)^{n+1}
$$

and

$$
C_{\hat{\gamma}}^{+}=\min \left(\frac{1}{2}, \frac{1-\hat{\gamma}-2 n k_{(n+1)} \cdot\left|\sup _{\Omega \times \mathbb{R}} H\right| \cdot\left(\tilde{R}_{+}\right)^{n}}{3 \hat{\gamma}+1}\right) .
$$


3. $L^{1}$-norm of $|D u|$ in terms of the $L^{1}$-norm of $\left(u-\inf _{\Omega} u\right)$ or $\left(\sup _{\Omega} u-u\right)$. An initial stage. Assume that there exists a nonnegative constant $H_{*}$ such that

$$
|H(x, t)| \leq H_{*} \text { for } x \in \Omega \text { and } t \in \mathbb{R} .
$$

Consider the identity (0.7). Assuming $|u|$ is bounded up to the boundary, [8, Theorem 1] assures us of that $u \in H^{1,1}(\Omega)$ and thus we are allowed to set in $(0.7)$

$$
\eta(x)=u(x)-\inf _{\Omega} u \geq 0
$$

and obtain

$$
\int_{\Omega} \frac{|D u|^{2}}{1+|D u|^{2}} d x-H_{*} \cdot \int_{\Omega}\left(u(x)-\inf _{\Omega} u\right) d x \leq \int_{\partial \Omega} \beta \cdot\left(u(x)-\inf _{\Omega} u\right) d \mathcal{H}_{n-1} .
$$

Since

the last inequality yields

$$
\frac{|D u|^{2}}{\sqrt{1+|D u|^{2}}}=\sqrt{1+|D u|^{2}}-\frac{1}{\sqrt{1+|D u|^{2}}},
$$

$$
\begin{aligned}
\int_{\Omega} \sqrt{1+|D u|^{2}} d x \leq|\Omega|+H_{*} & \cdot \int_{\Omega}\left(u(x)-\inf _{\Omega} u\right) d x \\
& +\int_{\partial \Omega \cap\{x: \beta(x)>0\}} \beta(x) \cdot\left(u(x)-\inf _{\Omega} u\right) d \mathcal{H}_{n-1} .
\end{aligned}
$$

Analogously, we are allowed to set in (0.7)

$$
\eta(x)=u(x)-\sup _{\Omega} u \leq 0,
$$

and obtain

$$
\begin{aligned}
\int_{\Omega} \sqrt{1+|D u|^{2}} d x \leq|\Omega|+H_{*} \cdot \int_{\Omega}\left(\sup _{\Omega} u-u(x)\right) d x \\
-\int_{\partial \Omega \cap\{x: \beta(x)<0\}} \beta(x) \cdot\left(\sup _{\Omega} u-u(x)\right) d \mathcal{H}_{n-1} .
\end{aligned}
$$

3.1. Restricting to small domains. This consideration suggests that we should restrict our consideration to a small region $\Omega \cap A_{0}$ of the type indicated below. Namely, setting

$$
\partial^{*} \Omega_{t}=\{x: x \in \Omega, \operatorname{dist}(x, \partial \Omega)=t\} \text { for } t>0,
$$

we first let the boundary $\partial\left(\Omega \cap A_{0}\right)$ be made up of three parts, namely

$$
\partial\left(\Omega \cap A_{0}\right)=\left(\partial \Omega \cap \overline{A_{0}}\right) \cup\left(\partial^{*} A_{0}\right) \cup\left(\partial^{* *} A_{0}\right),
$$

such that

$$
\partial^{*} A_{0}=\partial A_{0} \cap \partial^{*} \Omega_{\delta_{0}}
$$


for some small positive number $\delta_{0}$,

$$
\partial^{* *} A_{0}=\left(\partial A_{0} \cap \Omega\right) \backslash \partial^{*} \Omega_{\delta_{0}},
$$

and

$$
\left.D d \cdot \nu_{\Omega \cap A_{0}}\right|_{\partial^{* *} A_{0}}=0
$$

where we let $\nu_{\Omega \cap A_{0}}$ be the unit outward normal to $\partial\left(\Omega \cap A_{0}\right)$; furthermore,

$$
\begin{array}{ccc}
\operatorname{diam}\left(\partial \Omega \cap \overline{A_{0}}\right) \leq\left(\delta_{0}\right)^{1+\varepsilon_{0}} \quad \text { and } & \operatorname{diam}\left(\partial^{*} A_{0}\right) \leq\left(\delta_{0}\right)^{1+\varepsilon_{0}}, \\
\left|\partial \Omega \cap \overline{A_{0}}\right| \geq\left(\frac{\delta_{0}}{2}\right)^{\left(1+\varepsilon_{0}\right)(n-1)} & \text { and } & \left|\partial^{*} A_{0}\right| \geq\left(\frac{\delta_{0}}{2}\right)^{\left(1+\varepsilon_{0}\right)(n-1)},
\end{array}
$$

for some small positive constant $\varepsilon_{0}<1$. We choose $\delta_{0}$ sufficiently small so that each component of $\partial^{* *} A_{0}$ is entirely included in either $\partial_{+}^{* *}\left(\Omega \cap A_{0}\right)$ or $\partial_{-}^{* *}\left(\Omega \cap A_{0}\right)$, where

$$
\begin{aligned}
& \partial_{-}^{* *}\left(\Omega \cap A_{0}\right)=\left(\partial^{* *} A_{0}\right) \cap\left\{x: \beta_{\Omega \cap A_{0}}<0\right\} \\
& \partial_{+}^{* *}\left(\Omega \cap A_{0}\right)=\left(\partial^{* *} A_{0}\right) \cap\left\{x: \beta_{\Omega \cap A_{0}}>0\right\} .
\end{aligned}
$$

Next, we let the region $\Omega \cap A$ be as follows. Namely,

Case 1. If

$$
\left.\beta_{\Omega \cap A_{0}}\right|_{\partial^{* *} A_{0}} \leq-\hat{\beta} \quad \text { or }\left.\quad \beta_{\Omega \cap A_{0}}\right|_{\partial^{* *} A_{0}} \geq \hat{\beta}
$$

for some positive constant $\hat{\beta}$, then we let $A=A_{0}$.

Case 2. Suppose both sets $\partial_{-}^{* *}\left(\Omega \cap A_{0}\right)$ and $\partial_{-}^{* *}\left(\Omega \cap A_{0}\right)$ are nonempty and there hold

$$
\left.\beta_{\Omega \cap A_{0}}\right|_{\partial_{-}^{* *}\left(\Omega \cap A_{0}\right)} \leq-\hat{\beta} \text { and }\left.\beta_{\Omega \cap A_{0}}\right|_{\partial_{+}^{* *}\left(\Omega \cap A_{0}\right)} \geq \hat{\beta}
$$

For

$$
\beta(x)>\tilde{\tilde{\beta}}>0 \text { for all } x \in \partial \Omega \cap \overline{A_{0}}
$$

we set

$$
\begin{aligned}
& E_{+-}=\left\{x: x \in \overline{\Omega_{\delta_{0}}}, u(x)=\inf _{\partial \Omega \cap \overline{A_{0}}} u\right\}, \\
& E_{-+}=\left\{x: x \in \overline{\Omega_{\delta_{0}}}, u(x)=\sup _{\partial^{*} A_{0}} u\right\},
\end{aligned}
$$

and let $A_{11}$ be the region enclosed by $\partial^{*} \Omega_{\delta_{0}}$ and $E_{+-}$, together with the components of $\overline{\partial^{* *} A_{0}}$ passing through $E_{+-} \cap \partial \Omega$; let $A_{12}$ be the region enclosed by $\partial^{*} \Omega_{\delta_{0}}$ and $E_{-+}$, together with the components of $\overline{\partial^{* *} A_{0}}$ passing through $E_{-+} \cap \partial \Omega$; for

$$
\beta(x)<-\tilde{\tilde{\beta}}<0 \quad \text { for all } x \in \partial \Omega \cap \overline{A_{0}}
$$

we set

$$
\begin{aligned}
& E_{++}=\left\{x: x \in \overline{\Omega_{\delta_{0}}}, u(x)=\sup _{\partial \Omega \cap \overline{A_{0}}} u\right\}, \\
& E_{--}=\left\{x: x \in \overline{\Omega_{\delta_{0}}}, u(x)=\inf _{\partial^{*} A_{0}} u\right\}
\end{aligned}
$$


and let $A_{11}$ be the region enclosed by $\partial^{*} \Omega_{\delta_{0}}$ and $E_{++}$, together with components of $\overline{\partial^{* *} A_{0}}$ passing through $E_{++} \cap \partial \Omega$, and let $A_{12}$ be the region enclosed by $\partial^{*} \Omega_{\delta_{0}}$ and $E_{--}$, together with components of $\overline{\partial^{* *} A_{0}}$ passing through $E_{--} \cap \partial \Omega$. We then denote

$$
A=A_{0} \cup A_{11} \cup A_{12} .
$$

Furthermore, we let $\delta_{0}$ be so small that in the case of (1.15) or (1.16), there hold respectively

$$
-(1+\tilde{\beta}) / 2<\left.\beta_{\Omega \cap A}\right|_{\partial^{*} A}<-\tilde{\tilde{\beta}} / 2, \quad \text { or } \quad(1+\tilde{\beta}) / 2>\left.\beta_{\Omega \cap A}\right|_{\partial^{*} A}>\tilde{\tilde{\beta}} / 2,
$$

where we set

$$
\partial^{*} A=A \cap \partial^{*} \Omega_{\delta_{0}} .
$$

We shall prove in Appendix 5 the following.

Proposition 3. Suppose that

$$
\lim _{x_{k} \rightarrow x_{0}}|D u| \geq \frac{2}{\tilde{\beta}}
$$

for $\underline{\text { each }}$ sequence of points $x_{k}$ approaching $x_{0}$. Then we have

$$
\left|E_{ \pm \pm}\right| \leq 2\left|\partial^{* *} A\right| \cdot\left(\sqrt{1-\left\{(1+\tilde{\beta}) / 2 / \sqrt{1+[(1+\tilde{\beta}) / 2]^{2}}\right\}}\right)^{-1}
$$

and

$$
\left|A_{1}\right| \leq 2\left|\partial^{* *} A\right|^{2} \cdot \frac{(1+\tilde{\beta}) / 2 / \sqrt{1+[(1+\tilde{\beta}) / 2]^{2}}}{\left.\sqrt{1-\left\{(1+\tilde{\beta}) / 2 / \sqrt{1+[(1+\tilde{\beta}) / 2]^{2}}\right.}\right\}}
$$

For a domain $\Omega \cap A$, we may, without loss of generality, assume that

$$
\int_{\partial(\Omega \cap A)} \beta_{\Omega \cap A}(x) u(x) d \mathcal{H}_{n-1}=0
$$

where

$$
\beta_{\Omega \cap A}=\frac{D u}{\sqrt{1+|D u|^{2}}} \cdot \nu_{\Omega \cap A} .
$$

Applying to $\Omega \cap A$ the reasoning leading to (1.7) and (1.8), we obtain from (1.21)

$$
\begin{aligned}
\int_{\Omega \cap A}|D u| d x \leq|\Omega \cap A|+H_{*} \cdot \int_{\Omega \cap A} & \left(u(x)-\inf _{\Omega \cap A} u\right) d x \\
& +\int_{\partial(\Omega \cap A)} \beta_{\Omega \cap A} \cdot\left(u(x)-\inf _{\Omega \cap A} u\right) d \mathcal{H}_{n-1}
\end{aligned}
$$

and 


$$
\begin{aligned}
\int_{\Omega \cap A}|D u| d x \leq|\Omega \cap A|+H_{*} \cdot \int_{\Omega \cap A} & \left(\sup _{\Omega \cap A} u-u(x)\right) d x \\
& +\int_{\partial(\Omega \cap A)} \beta_{\Omega \cap A} \cdot\left(\sup _{\Omega \cap A} u-u(x)\right) d \mathcal{H}_{n-1} .
\end{aligned}
$$

Adding (1.22) and (1.23), we obtain

$$
\begin{aligned}
2 \int_{\Omega \cap A}|D u| d x \leq & |\Omega \cap A|+H_{*} \cdot \int_{\Omega \cap A}\left(u(x)-\inf _{\Omega \cap A} u\right) d x+H_{*} \cdot \int_{\Omega \cap A}\left(\sup _{\Omega \cap A} u-u(x)\right) d x \\
& +2 \int_{\partial(\Omega \cap A)} \beta(x) u(x) d \mathcal{H}_{n-1}-\left(\sup _{\Omega \cap A} u-\inf _{\Omega \cap A} u\right) \cdot \int_{\partial(\Omega \cap A)} \beta(x) d \mathcal{H}_{n-1} .
\end{aligned}
$$

This and (1.21) yield

$$
\begin{aligned}
2 \int_{\Omega \cap A}|D u| d x \leq & |\Omega \cap A|+H_{*} \cdot \int_{\Omega \cap A}\left(u(x)-\inf _{\Omega \cap A} u\right) d x+H_{*} \cdot \int_{\Omega \cap A}\left(\sup _{\Omega \cap A} u-u(x)\right) d x \\
& -\left(\sup _{\Omega \cap A} u-\inf _{\Omega \cap A} u\right) \cdot \int_{\partial(\Omega \cap A)} \beta(x) d \mathcal{H}_{n-1} .
\end{aligned}
$$

3.2. Boundary integral in (1.24). Taking $\eta=1$ in the identity (0.7) with the domain of integration $\Omega$ replaced by $\Omega \cap A$, we obtain

$$
\int_{\Omega \cap A} H d x=\int_{\Omega \cap A} \operatorname{div} \frac{D u}{\sqrt{1+|D u|^{2}}} d x=\int_{\partial(\Omega \cap A)} \beta_{\Omega \cap A}(x) d \mathcal{H}_{n-1} .
$$

Hence

$$
\left|\int_{\partial(\Omega \cap A)} \beta_{\Omega \cap A}(x) d \mathcal{H}_{n-1}\right| \leq H_{*} \cdot|\Omega \cap A| .
$$

3.3. Inserting (1.25) into (1.24), we obtain

$$
\begin{aligned}
2 \int_{\Omega \cap A}|D u| d x \leq & |\Omega \cap A|+H_{*} \cdot \int_{\Omega \cap A}\left(u(x)-\inf _{\Omega \cap A} u\right) d x+H_{*} \cdot \int_{\Omega \cap A}\left(\sup _{\Omega \cap A} u-u(x)\right) d x \\
& +\left(\sup _{\Omega \cap A} u-\inf _{\Omega \cap A} u\right) \cdot H_{*} \cdot|\Omega \cap A| .
\end{aligned}
$$

4. Estimating the $\mathbf{L}^{1}$-norm of $\left(u(x)-\inf _{\Omega \cap A} u\right)$ and $\left(\sup _{\Omega \cap A} u-u(x)\right)$ in $\Omega \cap A$.

4.1. $\mathbf{L}^{1}$-norm of $\left(\sup _{\Omega \cap A} u-u(x)\right)$ and $\left(u(x)-\inf _{\Omega \cap A} u\right)$ in $\Omega \cap A$. The case where $\beta(x)>0$ for all $x \in \partial \Omega \cap \bar{A}$. By the modified Sobolev inequality (A.8), we have

$$
\left\|\sup _{\Omega \cap A} u-u(x)\right\|_{L^{n_{*}}(\Omega \cap A)} \leq \frac{\omega_{n}}{n} \cdot\left[\int_{\Omega \cap A}|D u| d x+\int_{\partial(\Omega \cap A)}\left(\sup _{\Omega \cap A} u-u(x)\right) d \mathcal{H}_{n-1}\right] .
$$


Assume that (1.15) and (1.17) hold. By (A.2) and (A.7), we have

$$
\begin{aligned}
\int_{\partial^{*} A}\left(\sup _{\Omega \cap A} u-u(x)\right) d \mathcal{H}_{n-1} \leq \int_{\Omega \cap A}|D u| d x \\
+\left[2(n-1) \mathcal{K}_{\partial \Omega}+2\left(\delta_{0}\right)^{-1}\right] \cdot \int_{\Omega \cap A}\left(\sup _{\Omega \cap A} u-u(x)\right) d x
\end{aligned}
$$

and

$$
\begin{aligned}
\int_{\partial \Omega \cap \bar{A}}\left(\sup _{\Omega \cap A} u-u(x)\right) d \mathcal{H}_{n-1} \leq \int_{\Omega \cap A}|D u| d x \\
+\left[2(n-1) \mathcal{K}_{\partial \Omega}+2\left(\delta_{0}\right)^{-1}\right] \cdot \int_{\Omega \cap A}\left(\sup _{\Omega \cap A} u-u(x)\right) d x .
\end{aligned}
$$

Consider, rather than (1.6), the identity

$$
\int_{\Omega \cap A} \frac{D u}{\sqrt{1+|D u|^{2}}} \cdot D \eta d x+\int_{\Omega \cap A} H \cdot \eta d x-\int_{\partial(\Omega \cap A)} \beta_{\Omega \cap A} \cdot \eta d \mathcal{H}_{n-1}=0,
$$

for all $\eta \in H^{1,1}(\Omega \cap A)$. By setting $\eta(x)=\left(u(x)-\sup _{\Omega \cap A} u\right)$ and $\eta(x)=\left(\sup _{\Omega \cap A} u-\right.$ $u(x))$ in (1.30) we have

$$
\begin{gathered}
\int_{\Omega \cap A} \frac{|D u|^{2}}{\sqrt{1+|D u|^{2}}} d x-\int_{\Omega \cap A} H \cdot\left(\sup _{\Omega \cap A} u-u(x)\right) d x \\
+\int_{\partial(\Omega \cap A)} \beta_{\Omega \cap A} \cdot\left(\sup _{\Omega \cap A} u-u(x)\right) d \mathcal{H}_{n-1}=0
\end{gathered}
$$

and

$$
\begin{gathered}
\int_{\Omega \cap A} \frac{|D u|^{2}}{\sqrt{1+|D u|^{2}}} d x+\int_{\Omega \cap A} H \cdot\left(\sup _{\Omega \cap A} u-u(x)\right) d x \\
-\int_{\partial(\Omega \cap A)} \beta_{\Omega \cap A} \cdot\left(\sup _{\Omega \cap A} u-u(x)\right) d \mathcal{H}_{n-1}=0 .
\end{gathered}
$$

These yield

$$
\begin{aligned}
& \quad-\int_{\partial_{-}^{* *}(\Omega \cap A)} \beta_{\Omega \cap A} \cdot\left(\sup _{\Omega \cap A} u-u(x)\right) d \mathcal{H}_{n-1}-\int_{\partial^{*} A} \beta_{\Omega \cap A} \cdot\left(\sup _{\Omega \cap A} u-u(x)\right) d \mathcal{H}_{n-1} \\
& \leq \int_{\Omega \cap A} \frac{|D u|^{2}}{\sqrt{1+|D u|^{2}}} d x-\int_{\Omega \cap A} H \cdot\left(\sup _{\Omega \cap A} u-u(x)\right) d x \\
& \quad+\int_{\partial \Omega \cap \bar{A}} \beta_{\Omega \cap A} \cdot\left(\sup _{\Omega \cap A} u-u(x)\right) d \mathcal{H}_{n-1}
\end{aligned}
$$




$$
+\int_{\partial_{+}^{* *}(\Omega \cap A)} \beta_{\Omega \cap A} \cdot\left(\sup _{\Omega \cap A} u-u(x)\right) d \mathcal{H}_{n-1},
$$

and

$$
\begin{aligned}
& \int_{\partial_{+}^{* *}(\Omega \cap A)} \beta_{\Omega \cap A} \cdot\left(\sup _{\Omega \cap A} u-u(x)\right) d \mathcal{H}_{n-1}+\int_{\partial \Omega \cap \bar{A}} \beta_{\Omega \cap A} \cdot\left(\sup _{\Omega \cap A} u-u(x)\right) d \mathcal{H}_{n-1} \\
\leq & \int_{\Omega \cap A} \frac{|D u|^{2}}{\sqrt{1+|D u|^{2}}} d x+\int_{\Omega \cap A} H \cdot\left(\sup _{\Omega \cap A} u-u(x)\right) d x \\
& -\int_{\partial^{*} A} \beta_{\Omega \cap A} \cdot\left(\sup _{\Omega \cap A} u-u(x)\right) d \mathcal{H}_{n-1} \\
& -\int_{\partial_{-}^{* *} \Omega \cap A} \beta_{(\Omega \cap A)} \cdot\left(\sup _{\Omega \cap A} u-u(x)\right) d \mathcal{H}_{n-1},
\end{aligned}
$$

where we set

$$
\begin{aligned}
& \partial_{-}^{* *}(\Omega \cap A)=\left(\partial^{* *} A\right) \cap\left\{x: \beta_{\Omega \cap A}<0\right\} \\
& \partial_{+}^{* *}(\Omega \cap A)=\left(\partial^{* *} A\right) \cap\left\{x: \beta_{\Omega \cap A}>0\right\}
\end{aligned}
$$

4.1.1. If (1.13) holds for some positive constant $\hat{\beta}$, then we have $A=A_{0}$ and we obtain from (1.31) or (1.32)

$$
\begin{aligned}
\int_{\partial^{* *} A}\left(\sup _{\Omega \cap A} u-u(x)\right) d \mathcal{H}_{n-1} & \leq(\hat{\beta})^{-1} \cdot\left[\int_{\Omega \cap A}|D u| d x+H_{*} \int_{\Omega \cap A} \cdot\left(\sup _{\Omega \cap A} u-u(x)\right) d x\right] \\
& +(\hat{\beta})^{-1} \cdot \int_{\partial \Omega \cap \bar{A}}\left(\sup _{\Omega \cap A} u-u(x)\right) d \mathcal{H}_{n-1} .
\end{aligned}
$$

Inserting (1.29) into this, we obtain

$$
\begin{aligned}
& \int_{\partial^{* *} A}\left(\sup _{\Omega \cap A} u-u(x)\right) d \mathcal{H}_{n-1} \leq 2(\hat{\beta})^{-1} \cdot \int_{\Omega \cap A}|D u| d x \\
& \left.\quad+(\hat{\beta})^{-1}\left[H_{*}+2(n-1) \mathcal{K}_{\partial \Omega}+2\left(\delta_{0}\right)^{-1}\right] \cdot \int_{\Omega \cap A}\left(\sup _{\Omega \cap A} u-u(x)\right) d x\right] .
\end{aligned}
$$

Inserting (1.33), (1.28) and (1.29) into (1.27), we obtain

$$
\begin{aligned}
\left\|\sup _{\Omega \cap A} u-u(x)\right\|_{L^{n_{*}(\Omega \cap A)} \leq} & \frac{\omega_{n}}{n} \cdot\left[1+2(\hat{\beta})^{-1}\right] \cdot \int_{\Omega \cap A}|D u| d x \\
& +\hat{C}_{1} \cdot \int_{\Omega \cap A}\left(\sup _{\Omega \cap A} u-u(x)\right) d x,
\end{aligned}
$$


where

$$
\hat{C}_{1}=2(\hat{\beta})^{-1} \cdot H_{*}+\left[1+2(\hat{\beta})^{-1}\right] \cdot\left[2(n-1) \mathcal{K}_{\partial \Omega}+2\left(\delta_{0}\right)^{-1}\right] .
$$

Hence, by Hölder's inequality and the fact that $A=A_{0}$, we obtain

$$
\begin{aligned}
\int_{\Omega \cap A}|D u| d x \geq & {\left[1+2(\hat{\beta})^{-1}\right]^{-1} \cdot\left[n / \omega_{n}-\hat{C}_{1} \cdot\left|\Omega \cap A_{0}\right|^{1 / n}\right] } \\
& \times\left\|\sup _{\Omega \cap A} u-u(x)\right\|_{L^{n *}(\Omega \cap A)} .
\end{aligned}
$$

Analogously, we can establish in the case of (1.13) that

$$
\begin{aligned}
\int_{\Omega \cap A}|D u| d x \geq & {\left[1+2(\hat{\beta})^{-1}\right]^{-1} \cdot\left[n / \omega_{n}-\hat{C}_{1} \cdot\left|\Omega \cap A_{0}\right|^{1 / n}\right] } \\
& \times\left\|u(x)-\inf _{\Omega \cap A} u\right\|_{L^{n_{*}(\Omega \cap A} .}
\end{aligned}
$$

4.1.2. Suppose both sets $\partial_{-}^{* *}\left(\Omega \cap A_{0}\right)$ and $\partial_{-}^{* *}\left(\Omega \cap A_{0}\right)$ are nonempty and (1.14) holds. We notice that by our choice of $A_{0}$ and $A$ indicated above in (1.14) and (1.15), we have

$$
\sup _{\Omega \cap A} u=\sup _{\partial \Omega \cap \overline{A_{0}}} u \quad \text { and } \inf _{\Omega \cap A} u=\inf _{\partial^{*} A_{0}} u
$$

Thus

$$
\begin{aligned}
\int_{\partial(\Omega \cap A)}\left(\sup _{\Omega \cap A} u-u(x)\right) d \mathcal{H}_{n-1} \\
\quad=\int_{\partial(\Omega \cap A)}\left(\inf _{\partial \Omega \cap \overline{A_{0}}} u-u(x)\right) d \mathcal{H}_{n-1}+\int_{\partial(\Omega \cap A)}\left(\sup _{\partial \Omega \cap \overline{A_{0}}} u-\inf _{\partial \Omega \cap \overline{A_{0}}} u\right) d \mathcal{H}_{n-1} .
\end{aligned}
$$

By Theorem 1, we obtain

$$
\begin{aligned}
& \quad \int_{\partial(\Omega \cap A)} \beta_{\Omega \cap A} \cdot\left(\sup _{\partial \Omega \cap \overline{A_{0}}} u-\inf _{\partial \Omega \cap \overline{A_{0}}} u\right) d \mathcal{H}_{n-1} \mid \\
& \leq L_{0}(\tilde{\beta}, \tilde{\tilde{\beta}}) \cdot \operatorname{diam}\left(\partial \Omega \cap \overline{A_{0}}\right) \cdot|\partial(\Omega \cap A)|,
\end{aligned}
$$

where $L_{0}(\tilde{\beta}, \tilde{\tilde{\beta}})$ is the Lipschitz norm of the trace of $u$ on the boundary, which depends only on $\tilde{\beta}, \tilde{\tilde{\beta}}, H, n$ and $\mathcal{K}_{\partial \Omega \cap \bar{A}}$.

To treat the first integral on the right-hand side of (1.32), we set

$$
(\Omega \cap A)_{+-}=\left\{x: x \in \Omega \cap A, u(x) \leq \inf _{\partial \Omega \cap \overline{A_{0}}} u\right\},
$$

and

$$
\partial_{+-}^{* *}(\Omega \cap A)=\partial\left((\Omega \cap A)_{+-}\right) \cap \partial^{* *} A
$$

to obtain

$$
\int_{\left(\partial \Omega \cap \overline{A_{0}}\right) \cup \partial_{+}^{* *}(\Omega \cap A)}\left(\inf _{\partial \Omega \cap \bar{A}} u-u(x)\right) d \mathcal{H}_{n-1} \leq \int_{\partial_{+-}^{* *}(\Omega \cap A)}\left(\inf _{\partial \Omega \cap \bar{A}} u-u(x)\right) d \mathcal{H}_{n-1}=0,
$$


since $\partial_{+-}^{* *}(\Omega \cap A) \subset E_{+-}$. Furthermore, we have

$$
\begin{aligned}
& \int_{\partial \Omega \cap\left(\bar{A} \backslash \overline{A_{0}}\right)}\left(\inf _{\partial \Omega \cap \overline{A_{0}}} u\right.-u(x)) d \mathcal{H}_{n-1} \\
&\left.\leq L_{0}(\tilde{\beta}, \tilde{\tilde{\beta}}) \cdot \operatorname{diam}\left(\partial \Omega \cap \bar{A} \backslash \overline{A_{0}}\right)\right) \cdot\left|\partial \Omega \cap\left(\bar{A} \backslash \overline{A_{0}}\right)\right|
\end{aligned}
$$

and

$$
\int_{\partial^{*} A \cup \partial_{-}^{* *} A}\left(\inf _{\partial \Omega \cap \bar{A}_{0}} u-u(x)\right) d \mathcal{H}_{n-1} \leq \int_{\partial\left(\cap(\Omega \cap A)_{+-}\right)}\left(\inf _{\partial \Omega \cap \overline{A_{0}}} u-u(x)\right) d \mathcal{H}_{n-1},
$$

and

$$
\begin{aligned}
& -\int_{\partial\left(\cap(\Omega \cap A)_{+-}\right)} \beta_{\Omega \cap A}(x) \cdot\left(\inf _{\partial \Omega \cap \bar{A}_{0}} u-u(x)\right) d \mathcal{H}_{n-1} \\
\leq & \int_{(\Omega \cap A)_{+-}} \frac{|D u|^{2}}{\sqrt{1+|D u|^{2}}} d x+H_{*} \int_{(\Omega \cap A)_{+-}}\left(\inf _{\partial \Omega \cap \bar{A}} u-u(x)\right) d x \\
\leq & \int_{(\Omega \cap A)_{+-}} \frac{|D u|^{2}}{\sqrt{1+|D u|^{2}}} d x+H_{*} \int_{(\Omega \cap A)_{+-}}\left(\sup _{\Omega \cap A} u-u(x)\right) d x .
\end{aligned}
$$

From (1.39) and (1.40), we obtain

$$
\begin{aligned}
& \int_{\partial^{*} A \cup \partial_{-}^{* * A}}\left(\inf _{\partial \Omega \cap \overline{A_{0}}} u-u(x)\right) d \mathcal{H}_{n-1} \\
& \quad \leq(\hat{\hat{\beta}})^{-1} \cdot \int_{(\Omega \cap A)_{+-}}|D u| d x+(\hat{\hat{\beta}})^{-1} \cdot H_{*} \cdot \int_{(\Omega \cap A)_{+-}}\left(\inf _{\partial \Omega \cap \overline{A_{0}}} u-u(x) d x\right.
\end{aligned}
$$

if we have

$$
\left.\left|\beta_{\Omega \cap A}\right|\right|_{\partial_{-}^{* *} A} \geq \hat{\hat{\beta}}_{*}
$$

and we set

$$
\hat{\hat{\beta}}=\min \left(\hat{\hat{\beta}}_{*}, \tilde{\tilde{\beta}} / 2\right) \text {. }
$$

Inserting (1.36), (1.37), (1.38) and (1.41) into (1.27) and using Hölder's inequality, we obtain the inequality

$$
\begin{aligned}
& \left(1+(\hat{\hat{\beta}})^{-1}\right) \cdot \int_{\Omega \cap A}|D u| d x+2 L_{0}(\tilde{\beta}, \tilde{\tilde{\beta}}) \cdot \operatorname{diam}(\partial \Omega \cap \bar{A}) \cdot|\partial(\Omega \cap A)| \\
& \geq \frac{n}{\omega_{n}}\left(1-(\hat{\hat{\beta}})^{-1} \cdot \frac{\omega_{n}}{n} \cdot H_{*} \cdot|\Omega \cap A|^{1 / n}\right) \cdot\left\|\sup _{\Omega \cap A} u-u(x)\right\|_{L^{n *}(\Omega \cap A)}
\end{aligned}
$$

if (1.42.1) holds and $\hat{\hat{\beta}}$ is given by (1.42.2). 
4.1.3. Analogously, we can use (1.17) and Theorem 1 to establish

$$
\begin{aligned}
& \left(1+(\hat{\hat{\beta}})^{-1}\right) \cdot \int_{\Omega \cap A}|D u| d x+2 L_{0}((1+\tilde{\beta}) / 2, \tilde{\tilde{\beta}} / 2) \cdot \operatorname{diam}\left(\partial^{*} A\right) \cdot|\partial(\Omega \cap A)| \\
& \geq \frac{n}{\omega_{n}} \cdot\left(1-(\hat{\hat{\beta}})^{-1} \cdot \frac{\omega_{n}}{n} \cdot H_{*} \cdot|\Omega \cap A|^{1 / n}\right) \cdot\left\|u(x)-\inf _{\Omega \cap A} u\right\|_{L^{n *}(\Omega \cap A},
\end{aligned}
$$

where the constant $L_{0}((1+\tilde{\beta}) / 2, \tilde{\tilde{\beta}} / 2)$ is given in Theorem 1 .

4.1.4. Inserting (1.35.1), (1.35.2), (1.43) and (1.44) into (1.26) and applying Hölder's inequality, we obtain

$$
\begin{aligned}
& \int_{\Omega \cap A}(\left.\sup _{\Omega \cap A} u-u(x)\right) d x+\int_{\Omega \cap A}\left(u(x)-\inf _{\Omega \cap A} u\right) d x \\
& \leq\left[\hat{C}_{2}+\left(1+(\hat{\hat{\beta}})^{-1}\right) \cdot \hat{C}_{3}\right] \cdot|\Omega \cap A|^{1+1 / n}+\left[\hat{C}_{2}+(\hat{\hat{\beta}})^{-1} \cdot \hat{C}_{3}\right] \\
& \quad \times|\Omega \cap A|^{1 / n} \cdot \int_{\Omega \cap A}\left(u(x)-\inf _{\Omega \cap A} u\right) d x \\
& \quad+\left[\hat{C}_{2}+(\hat{\hat{\beta}})^{-1} \cdot \hat{C}_{3}\right] \cdot|\Omega \cap A|^{1 / n} \cdot \int_{\Omega \cap A}\left(\sup _{\Omega \cap A} u-u(x)\right) d x \\
& \quad+\hat{C}_{3} \cdot L_{0}(\tilde{\beta}, \tilde{\tilde{\beta}}) \cdot \operatorname{diam}(\partial \Omega \cap \bar{A}) \cdot|\partial(\Omega \cap A)| \cdot|\Omega \cap A|^{1 / n}, \\
& \quad+\hat{C}_{3} \cdot L_{0}((1+\tilde{\beta}) / 2, \tilde{\tilde{\beta}} / 2) \cdot \operatorname{diam}\left(\partial^{*} A\right) \cdot|\partial(\Omega \cap A)| \cdot|\Omega \cap A|^{1 / n},
\end{aligned}
$$

where

$$
\begin{aligned}
\hat{C}_{2}= & \frac{2 \omega_{n}}{n}\left[1+3(\hat{\beta})^{-1}+(\hat{\hat{\beta}})^{-1}\right] \cdot\left[n / \omega_{n}-\hat{C}_{1} \cdot\left|\Omega \cap A_{0}\right|^{1 / n}\right]^{-1} \\
& +\frac{\omega_{n}}{n}\left(\sup _{\Omega \cap A} u-\inf _{\Omega \cap A} u\right) \cdot H_{*}, \\
\hat{C}_{3}= & \frac{\omega_{n}}{n} \cdot\left(1-(\hat{\hat{\beta}})^{-1} \cdot\left(\omega_{n} / n\right) \cdot H_{*} \cdot|\Omega \cap A|^{1 / n}\right)^{-1} .
\end{aligned}
$$

Hence

$$
\begin{aligned}
& \int_{\Omega \cap A}\left(\sup _{\Omega \cap A} u-u(x)\right) d x+\int_{\Omega \cap A}\left(u(x)-\inf _{\Omega \cap A} u\right) d x \\
\leq & {\left[\hat{C}_{2}+(\hat{\hat{\beta}})^{-1} \cdot \hat{C}_{3}\right] \cdot \hat{C}_{4} \cdot|\Omega \cap A|^{1+1 / n} } \\
& +\hat{C}_{3} \cdot \hat{C}_{4} \cdot L_{0}(\tilde{\beta}, \tilde{\tilde{\beta}}) \cdot \operatorname{diam}(\partial \Omega \cap \bar{A}) \cdot|\partial(\Omega \cap A)| \cdot|\Omega \cap A|^{1 / n} \\
& +\hat{C}_{3} \cdot \hat{C}_{4} \cdot L_{0}((1+\tilde{\beta}) / 2, \tilde{\tilde{\beta}} / 2) \cdot \operatorname{diam}\left(\partial^{*} A\right) \cdot|\partial(\Omega \cap A)| \cdot|\Omega \cap A|^{1 / n},
\end{aligned}
$$

where

$$
\hat{C}_{4}=\left(1-\left[\hat{C}_{2}+(\hat{\hat{\beta}})^{-1} \cdot \hat{C}_{3}\right] \cdot H_{*} \cdot|\Omega \cap A|^{1 / n}\right)^{-1}
$$


This yields

$$
\begin{aligned}
\int_{\Omega \cap A} & \left(\sup _{\Omega \cap A} u-u(x)\right) d x \leq\left[\hat{C}_{2}+(\hat{\hat{\beta}})^{-1} \cdot \hat{C}_{3}\right] \cdot \hat{C}_{4} \cdot|\Omega \cap A|^{1+1 / n} \\
& +\hat{C}_{3} \cdot \hat{C}_{4} \cdot L_{0}(\tilde{\beta}, \tilde{\tilde{\beta}}) \cdot \operatorname{diam}(\partial \Omega \cap \bar{A}) \cdot|\partial(\Omega \cap A)| \cdot|\Omega \cap A|^{1 / n} \\
& +\hat{C}_{3} \cdot \hat{C}_{4} \cdot L_{0}((1+\tilde{\beta}) / 2, \tilde{\tilde{\beta}} / 2) \cdot \operatorname{diam}\left(\partial^{*} A\right) \cdot|\partial(\Omega \cap A)| \cdot|\Omega \cap A|^{1 / n}
\end{aligned}
$$

and

$$
\begin{aligned}
\int_{\Omega \cap A} & \left(u(x)-\inf _{\Omega \cap A} u\right) d x \leq\left[\hat{C}_{2}+(\hat{\hat{\beta}})^{-1} \cdot \hat{C}_{3}\right] \cdot \hat{C}_{4} \cdot|\Omega \cap A|^{1+1 / n} \\
& +\hat{C}_{3} \cdot \hat{C}_{4} \cdot L_{0}(\tilde{\beta}, \tilde{\tilde{\beta}}) \cdot \operatorname{diam}(\partial \Omega \cap \bar{A}) \cdot|\partial(\Omega \cap A)| \cdot|\Omega \cap A|^{1 / n} \\
& +\hat{C}_{3} \cdot \hat{C}_{4} \cdot L_{0}((1+\tilde{\beta}) / 2, \tilde{\tilde{\beta}} / 2) \cdot \operatorname{diam}\left(\partial^{*} A\right) \cdot|\partial(\Omega \cap A)| \cdot|\Omega \cap A|^{1 / n},
\end{aligned}
$$

By (1.9), (1.11) and (1.12), we have

$$
\left|\Omega \cap A_{0}\right| \leq\left(\delta_{0}\right)^{(n-1) \cdot\left(1+\varepsilon_{0}\right)+1}
$$

which yields

$$
\left|\Omega \cap A_{0}\right|^{\frac{1}{n}} \leq\left(\delta_{0}\right)^{\left(1-\frac{1}{n}\right) \cdot\left(1+\varepsilon_{0}\right)+\frac{1}{n}}=\left(\delta_{0}\right)^{1+\varepsilon_{0}\left(1-\frac{1}{n}\right)} .
$$

In view of $(1.34),(1.45)$ and (1.47), we see that

$$
\hat{C}_{1} \cdot\left|\Omega \cap A_{0}\right|^{1 / n} \leq \mathfrak{C}_{1} \cdot\left(\delta_{0}\right)^{\varepsilon_{0}\left(1-\frac{1}{n}\right)} \leq \frac{1}{2} \quad \text { for } \quad \delta_{0} \quad \text { sufficiently small, }
$$

where $\mathfrak{C}_{1}$ is a constant depending only on $H_{*}, \hat{\beta}$ and $\mathcal{K}_{\partial \Omega}$.

If (1.18) holds for each sequence of points $x_{k}$ approaching $x_{0}$, then we obtain from (1.19) and (1.50)

$$
|\Omega \cap A| \leq \tilde{\mathfrak{C}}_{0}\left(\delta_{0}\right)^{n},
$$

where $\tilde{\mathfrak{C}}_{0}$ is determined by $\tilde{\beta}$ and $n$. Hence, in view of $(1.45)$,

$$
\hat{C}_{2} \cdot|\Omega \cap A|^{\frac{1}{n}} \leq \mathfrak{C}_{2} \cdot\left(\delta_{0}\right) \leq 1 /\left[2 H_{*}\left(\sup _{\Omega \cap A} u-\inf _{\Omega \cap A} u\right)\right]
$$

for $\delta_{0}$ sufficiently small, and, by (1.47),

$$
\hat{C}_{3} \leq 2, \quad \hat{C}_{4} \leq 2, \quad \text { for } \delta_{0} \text { sufficiently small, }
$$

where $\mathfrak{C}_{2}$ is a constant depending only on $H_{*}, \tilde{\beta}, \hat{\beta}$ and $n$.

We remark here that (1.49) will not be used in the next sections.

\section{2. $\mathbf{L}^{1}$-norm of $\left(\sup _{\Omega \cap A} u-u(x)\right)$ and $\left(u(x)-\inf _{\Omega \cap A} u\right)$ in $\Omega \cap A$. The Case} where $\beta(x)<0$ for all $x \in \partial \Omega \cap \bar{A}$. Assume (1.16) holds. The case where $\beta(x)<0$ can be treated in a similar way as the case $\beta(x) \geq 0$ : repeating the corresponding reasoning, in case (1.13) holds, we obtain (1.35.1) and (1.35.2), in case (1.14) holds, we obtain (1.43) and (1.44). Inserting (1.35.1), (1.35.2), (1.44) and (1.45) into (1.26), we obtain (1.48) and (1.49). 
We also remark here that in this setting, (1.48) will not used in the nect sections.

\section{Oscillation of $u$ near the boundary.}

5.1. The case where $1>\beta\left(x_{0}\right)>0$. Consider a point $x_{0} \in \partial \Omega$ such that for $\delta_{0}$ sufficiently small such there holds

$$
1>\tilde{\beta}>\beta(x)>\tilde{\tilde{\beta}}>0, \text { for } x \in \partial \Omega \cap B_{\delta_{0}}\left(x_{0}\right) .
$$

We assume, without loss of generality, $\delta_{0}$ to be sufficiently small that $\delta_{0} \leq R_{-}$, $R_{-}$being given in Proposition 1 .

Choose a boundary strip $A$ adjacent to $\partial \Omega \cap B_{\tilde{\delta}_{0}}\left(x_{0}\right), \tilde{\delta}_{0}=\left(\delta_{0}\right)^{1+\varepsilon_{0}}$, to be with of width $\delta_{0}$ and of the type indicated in the beginning of Subsection 3.1 such that (1.17) hold. We obtain from (1.56) that (1.13) or (1.14) hold with

$$
\hat{\beta}=\frac{\tau}{\sqrt{1+\tau^{2}}} \cdot \sqrt{1-[(1+\tilde{\beta}) / 2)]^{2}},
$$

where

$$
\tau=\sqrt{(\tilde{\tilde{\beta}} / 2)^{2} /\left(1-(\tilde{\tilde{\beta}} / 2)^{2}\right)}
$$

and (1.42.1) holds with

$$
\hat{\hat{\beta}}_{*}=\tilde{\tilde{\beta}} / 2
$$

for $\delta_{0}$ sufficiently small. We shall establish this for sufficiently small $\delta_{0}$ in Appendix 4 .

Let us set

$$
R=\left(\delta_{0} / 4\right)^{1+\varepsilon_{0}}
$$

and choose a boundary strip $A_{R}\left(x_{0}\right)$ adjacent to $\partial \Omega \cap B_{R}\left(x_{0}\right)$ to be of width $R$ and of the type indicated above in Theorem 2. From inserting (1.46) into (1.2) with the value $\hat{\beta}$ given in (1.57) and setting $\hat{\gamma}=0$ in (1.5), we obtain

$$
\begin{aligned}
& u\left(x_{0}\right)-\inf _{A_{R}\left(x_{0}\right)} u \leq \frac{2^{n+2}}{\alpha_{*} R^{n}} \cdot\left\{\left[\hat{C}_{2}+(\hat{\hat{\beta}})^{-1} \cdot \hat{C}_{3}\right] \cdot \hat{C}_{4} \cdot|\Omega \cap A|^{1+\frac{1}{n}}\right. \\
& +\hat{C}_{3} \cdot \hat{C}_{4} \cdot L_{0}(\tilde{\beta}, \tilde{\tilde{\beta}}) \cdot \operatorname{diam}(\partial \Omega \cap \bar{A}) \cdot|\partial(\Omega \cap A)| \cdot|\Omega \cap A|^{1 / n} \\
& \left.+\hat{C}_{3} \cdot \hat{C}_{4} \cdot L_{0}((1+\tilde{\beta}) / 2, \tilde{\tilde{\beta}} / 2) \cdot \operatorname{diam}\left(\partial^{*} A\right) \cdot|\partial(\Omega \cap A)| \cdot|\Omega \cap A|^{1 / n}\right\}+2 R \\
& \leq 2^{n+6} \cdot(n+1) \cdot k_{(n+1)} \cdot\left\{\left[\hat{C}_{2}+(\hat{\hat{\beta}})^{-1} \cdot \hat{C}_{3}\right] \cdot \hat{C}_{3} \cdot \frac{|\Omega \cap A|^{1+\frac{1}{n}}}{R^{n}}\right. \\
& \quad+\hat{C}_{3} \cdot \hat{C}_{4} \cdot L_{0}(\tilde{\beta}, \tilde{\tilde{\beta}}) \cdot \operatorname{diam}(\partial \Omega \cap \bar{A}) \cdot|\partial(\Omega \cap A)| \cdot|\Omega \cap A|^{1 / n} / R^{n} \\
& \left.\quad+\hat{C}_{3} \cdot \hat{C}_{4} \cdot L_{0}((1+\tilde{\beta}) / 2, \tilde{\tilde{\beta}} / 2) \cdot \operatorname{diam}\left(\partial^{*} A\right) \cdot|\partial(\Omega \cap A)| \cdot|\Omega \cap A|^{1 / n} / R^{n}\right\} \\
& +2 R .
\end{aligned}
$$


If (1.18) holds for each sequence of points $x_{k}$ approaching $x_{0}$, then we obtain from $(1.20)$

$$
\begin{aligned}
& \operatorname{diam}(\partial \Omega \cap \bar{A}) \cdot|\partial(\Omega \cap A)| \cdot|\Omega \cap A|^{1 / n} \leq \tilde{\mathfrak{C}}_{0}^{*} \cdot\left(\delta_{0}\right)^{1+\frac{1}{n}}, \\
& \operatorname{diam}\left(\partial^{*} A\right) \cdot|\partial(\Omega \cap A)| \cdot|\Omega \cap A|^{1 / n} \leq \tilde{\mathfrak{C}}_{0}^{*} \cdot\left(\delta_{0}\right)^{1+\frac{1}{n}}
\end{aligned}
$$

where $\tilde{\mathfrak{C}}_{0}^{*}$ is determined by $\tilde{\beta}$ and $n$. Inserting these, together with (1.17), (1.42.1), (1.53) into (1.61) and then using (1.45), (1.54), (1.55), (1.17), (1.42.1), (1.42.2), (1.57), (1.58), (1.59), we arrive at (0.13) with $\hat{\mathfrak{C}}$ determined by $\tilde{\beta}, \tilde{\beta}$, $H_{*}$ and $\mathcal{K}_{\partial \Omega \cap \overline{A_{\delta_{0}}\left(x_{0}\right)}}$.

5.2. The case where $-1<\beta\left(x_{0}\right)<0$. Consider a point $x_{0} \in \partial \Omega$ such that for $\delta_{0}$ sufficiently small there holds

$$
-1<-\tilde{\beta}<\beta(x)<-\tilde{\tilde{\beta}}<0, \quad \text { for } x \in \partial \Omega \cap B_{\delta_{0}}\left(x_{0}\right) .
$$

Choose $\delta_{0}$ to be sufficiently small such that $\delta_{0} \leq R_{+}, R_{+}$being given in Proposition 1. Choose a boundary strip $A_{0}$ adjacent to $\partial \Omega \cap B_{\tilde{\delta}_{0}}\left(x_{0}\right), \tilde{\delta}_{0}=\left(\delta_{0}\right)^{1+\varepsilon_{0}}$, to be of width $\delta_{0}$ and of the type as before. For $\tau$ given in (1.58), we obtain from (1.62) that (1.13) or (1.14) holds with $\hat{\hat{\beta}}$ given in (1.57), and (1.42.1) holds with $\hat{\hat{\beta}}$ given in (1.59), which will be established for sufficiently small $\delta_{0}$ in Appendix 4.

From inserting (1.49) into (1.4) with the value of $\hat{\hat{\beta}}$ given in (1.57) and setting $\hat{\gamma}=0$ in (1.5), we obtain, analogously to $\mathbf{5 . 1}$, the estimate $(0.14)$ with the same $\hat{\mathfrak{C}}$ as in $(0.13)$.

\section{Appendix 1. Boundary Integrals along a Piecewise $\mathrm{C}^{2}$ Boundary}

The proof of the following can be modified from that of [6, Lemma 1.1] in an obvious way.

Lemma A.1. Let $E$ be a Caccioppoli set in $\mathbb{R}^{n}$ and $\Gamma$ be a subset of $\partial E$ which is a $C^{2}$ manifold and $d(x)=\operatorname{dist}(x, \partial E)$ for $x \in E$. Let

$$
E_{\Gamma, t}=\{x: x \in E: \operatorname{dist}(x, \Gamma) \leq t\}, \quad \text { for } t>0 .
$$

Let $\varepsilon_{\Gamma}$ be so small that the function $d(x)$ be of the class $C^{2}$ in $E_{\Gamma, \varepsilon_{\Gamma}}$, and consider, for $0<\varepsilon^{\prime}<\varepsilon_{\Gamma}$, a domain $E_{\Gamma, \varepsilon_{\Gamma}^{*}}^{*}$,

$$
E_{\Gamma, \varepsilon^{\prime}} \subseteq E_{\Gamma, \varepsilon_{\Gamma}}^{*} \subseteq E_{\Gamma, \varepsilon_{\Gamma}},
$$

such that a portion of its boundary $\partial^{*} E_{\Gamma, \varepsilon_{\Gamma}}^{*} \subset E_{\Gamma, t} \backslash E_{\Gamma, \varepsilon^{\prime}}$, and on the remaining portion of its boundary in $\Omega$, we have

$$
\left.D d \cdot \nu\right|_{\left(\partial E_{\Gamma, \varepsilon_{\Gamma}}^{*} \cap \Omega\right) \backslash \partial^{*} E_{\Gamma, \varepsilon_{\Gamma}}^{*}}=0
$$


$\nu$ being the unit outward normal to $\partial E_{\Gamma, \varepsilon_{\Gamma}}^{*}$. Then, there exists a constant $C_{\Gamma, \varepsilon^{\prime}}$ depending only on $\Gamma$ and $\varepsilon^{\prime}$ such that the inequality

$$
\int_{\Gamma} w d \mathcal{H}_{n-1} \leq \int_{E_{\Gamma, \varepsilon_{\Gamma}}^{*}}|D w| d x+C_{\Gamma, \varepsilon^{\prime}} \cdot \int_{E_{\Gamma, \varepsilon_{\Gamma}}^{*}}|w| d x
$$

holds for all $w \in B V\left(E_{\Gamma, \varepsilon_{\Gamma}}\right)$. In fact, let $\eta_{\varepsilon^{\prime}}$ be a $C^{\infty}$ function with

$$
\left\{\begin{array}{l}
o \leq \eta_{\varepsilon^{\prime}} \leq 1 \\
\eta_{\varepsilon^{\prime}}=1 \text { on } \Gamma \\
\eta_{\varepsilon^{\prime}}=0 \text { in } E \backslash E_{\Gamma, \varepsilon^{\prime}}
\end{array}\right.
$$

then we can take

$$
C_{\Gamma, \varepsilon^{\prime}}=\sup _{E_{\Gamma, \varepsilon_{\Gamma}}^{*}}\left|\operatorname{div}\left(\eta_{\varepsilon^{\prime}} D d\right)\right|
$$

In order to apply Lemma A.1, we have to estimate the value of $C_{\Gamma, \varepsilon^{\prime}}$ in (A.4). For this, we formulate the following result which is well known and can be found, e.g., in [5, pp. 420-422].

Lemma A.2. Let $\Gamma \subseteq \partial E$ be of the class $C^{2}$ whose principal curvatures are bounded in the absolute value by $\mathcal{K}_{\Gamma}$. Then $d(x)=\operatorname{dist}(x, \Gamma)$ is of the class $C^{2}$ in $E_{\Gamma, \varepsilon_{\Gamma}}$, for $\varepsilon_{\Gamma} \leq \frac{1}{\mathcal{K}_{\Gamma}}$, where $E_{\Gamma, \varepsilon_{\Gamma}}$ is given in (A.1).

Furthermore, for points $\bar{x}$ in $E_{\Gamma, \varepsilon_{\Gamma}}, \varepsilon_{\Gamma} \leq \frac{1}{\mathcal{K}_{\Gamma}}$, define $\bar{y}=\bar{y}(\bar{x})$ to be the (unique) point of $\Gamma$ nearest to $\bar{x}$. Consider the special coordinate frame in which the $x_{n}$-axis is oriented along the inward normal to $\Gamma$ at $\bar{y}$ and the coordinates $x_{1}, \cdots, x_{n-1}$ lie along the principal directions of $\Gamma$ at the point $\bar{y}$. In this special coordinates, we have at $\bar{x}$,

$$
D d=(0, \cdots, 0,1)
$$

and

$$
D^{2} d=\operatorname{diagonal}\left[\frac{-k_{1}}{1-k_{1} d}, \cdots, \frac{-k_{n-1}}{1-k_{n-1} d}, 0\right]
$$

where $k_{1}, \cdots, k_{n-1}$ are the principal curvatures of $\Gamma$ at $\bar{y}$.

Inserting (A.5) and (A.6) into (A.3) and (A.4), we obtain the following.

Lemma A.3. Let $\Gamma \subseteq \partial E$ be of the class $C^{2}$ whose principal curvatures are bounded in the absolute value by $\mathcal{K}_{\Gamma}$. Then, for $\varepsilon_{\Gamma} \leq \frac{1}{\mathcal{K}_{\Gamma}}$ and for each $\delta$, $0<\delta \leq 1$, we can take in (A.4)

$$
C_{\Gamma, \varepsilon^{\prime}} \leq\left|D \eta_{\varepsilon^{\prime}}\right|+2(n-1) \mathcal{K}_{\Gamma} \leq\left(\frac{1+\delta}{\varepsilon^{\prime}}\right)+2(n-1) \mathcal{K}_{\Gamma}
$$




\section{Appendix 2. Modified Sobolev Inequality}

The following result is a special case of the so-called Friedrichs inequality and can be found, e.g., in [9, Theorem 6.5.7].

Proposition A.1. Suppose $E$ is a Caccioppoli set with piecewise Lipschitz continuous boundary. Then, for any $f \in B V(\Omega)$, the inequality

$$
\|f\|_{L^{n *}(E)} \leq \frac{n}{\omega_{n}}\left(\int_{E}|D f| d x+\int_{\partial E}|f| d \mathcal{H}_{n-1}\right)
$$

is valid, where $\omega_{n}$ is the Lebesgue measure of the $n$-dimensional unit ball.

\section{Appendix 3. A Proof of (0.16)}

Since $u$ is assumed to be bounded up to the boundary, [8, Theorem 1] implies $u \in H^{1,1}(\Omega)$. By this and the fact that the restriction $\left.u\right|_{\Omega \backslash \Omega_{\varepsilon}}$ is a minimizing function of the functional

$$
J(v)=\int_{\Omega} \sqrt{1+|D u|^{2}} d x+\int_{\partial \Omega_{\varepsilon} \cap \Omega}\left(T u \cdot \nu_{\Omega_{\varepsilon}}\right) u d \mathcal{H}_{n-1},
$$

with $\nu_{\Omega_{\varepsilon}}$ being the unit outward normal to $\Omega_{\varepsilon}$, we are allowed to set $\eta=1$ in the identities

$$
\int_{\Omega} \frac{D u}{\sqrt{1+|D u|^{2}}} \cdot D \eta d x+\int_{\Omega} H \cdot \eta d x=\int_{\partial \Omega} \beta \cdot \eta d \mathcal{H}_{n-1}
$$

and

$$
\int_{\Omega \backslash \Omega_{\varepsilon}} \frac{D u}{\sqrt{1+|D u|^{2}}} \cdot D \eta d x+\int_{\Omega \backslash \Omega_{\varepsilon}} H \cdot \eta d x=\int_{\Omega_{\Omega_{\varepsilon} \cap \Omega}}\left(T u \cdot \nu_{\Omega_{\varepsilon}}\right) \cdot \eta d \mathcal{H}_{n-1} ;
$$

(see $[5,(7.6)])$; here $\Omega_{\varepsilon}=\{x: x \in \Omega$, $\operatorname{dist}(x, \partial \Omega) \leq \varepsilon\}$ and $\varepsilon$ is sufficiently small. Subtracting (A.10) (with $\eta=\left.u\right|_{\Omega \backslash \Omega_{\varepsilon}}$ ) from (A.9) (with $\eta=u$ ), we obtain

$$
\int_{\Omega_{\varepsilon}} H d x=\int_{\partial \Omega} \beta \cdot u d \mathcal{H}_{n-1}-\int_{\partial \Omega_{\varepsilon}}\left(T u \cdot \nu_{\Omega \backslash \Omega_{\varepsilon}}\right) d \mathcal{H}_{n-1} .
$$

The left-hand side of the last identity approaches zero as $\varepsilon \rightarrow 0$, hence the same is true for the right-hand side of the last identity. Hence, a subsequence can be extracted from the sequence $\left\{T u \cdot \nu_{\Omega \backslash \Omega_{\varepsilon}}\right\}(x+D d)$, with $d(x)=\operatorname{dist}(x, \partial \Omega)$, which approaches $\beta(x)$ for almost every $x \in \partial \Omega$ as $\varepsilon \rightarrow 0$. This, together with the interior regularity of $u$, yields under the assumption (0.12) that if $\delta_{0}$ is sufficiently small, then

$$
\frac{1+\tilde{\beta}}{2} \geq\left|T u \cdot \nu_{\Omega_{\delta_{0}}}(x)\right| \geq \frac{\tilde{\tilde{\beta}}}{2},
$$

for $x \in \partial^{*} \Omega_{\varepsilon}, \varepsilon \leq \delta_{0}$, which is sufficiently close to $A_{\delta_{0}}\left(x_{0}\right)$. In particular, $(0.16)$ is proved. 


\section{Appendix 4. Proof of (1.13), (1.14) with the Value of $\hat{\beta}$ Given} BY (1.57). A ProOF OF (1.59).

We shall prove in Appendix 5 that points of $\partial^{*} A$ are close to $\partial^{*} A_{\delta_{0}}\left(x_{0}\right)$ for small $\delta_{0}$. The second inequality in (A.11) thus yields

$$
\left.\frac{|D u|}{\sqrt{1+|D u|^{2}}}\right|_{\partial^{*} A} \geq \frac{\tilde{\tilde{\beta}}}{2}
$$

from which we obtain

$$
\left.|D u|\right|_{\partial^{*} A} \geq \tau
$$

with $\tau$ given in (1.58). This and the first inequality in (A.11) yield (1.57).

Since $E_{ \pm \pm}$are level sets of $u$, the normal of $E_{ \pm \pm}$lies toward or opposite to the direction of that of $\frac{D u}{\|D u\|}$, and thus

$$
\left.\left|\beta_{\Omega \cap A}\right|\right|_{E_{ \pm \pm}}=\frac{D u}{\sqrt{1+|D u|^{2}}} \cdot \frac{D u}{|D u|}=\frac{|D u|}{\sqrt{1+|D u|^{2}}}
$$

from which by (1.61) it follows the second inequality in (A.11).

\section{Appendix 5. Proof of Proposition 2}

To prove Proposition 2, we assume without loss of generality that (1.15) holds. To show the inequality (1.19) for $E_{+-}$, we observe that the unit normal of $E_{+-}$is $\pm \frac{D u}{|D u|}$ and thus it suffices to show that for points $x$ in a sufficiently small neighborhood of $x_{0}$

$$
\left|\frac{D u}{|D u|}(x) \cdot \nu_{\Omega}\left(x_{0}\right)\right| \leq \frac{(1+\tilde{\beta}) / 2}{\sqrt{1+[(1+\tilde{\beta}) / 2]^{2}}},
$$

if (1.18) holds for each sequence of points $x_{k}$ approaching $x_{0}$. This follows from the second inequality in (A.11) and the inequality

$$
\frac{\sqrt{1+|D u|^{2}}}{|D u|}(x)=\sqrt{1+\frac{1}{|D u|^{2}}} \leq \sqrt{1+[(1+\tilde{\beta}) / 2]^{2}}
$$

for points $x$ in a sufficiently small neighborhood of $x_{0}$, which is obtained from the assumption that (1.18) holds for each sequence of points $x_{k}$ approaching $x_{0}$.

\section{REFERENCES}

1. R. Finn, Equilibrium capillary surfaces. Grundlehren der Mathematischen Wissenschaften [Fundamental Principles of Mathematical Sciences], 284. Springer-Verlag, New York, 1986.

2. C. Gerhardt, Existence and regularity of capillary surfaces. Boll. Un. Mat. Ital. (4) 10(1974), 317-335.

3. C. Gerhardt, On the capillarity problem with constant volume. Ann. Scuola Norm. Sup. Pisa Cl. Sci. (4) 2(1975), No. 2, 303-320.

4. C. Gerhardt, Existence, regularity, and boundary behavior of generalized surfaces of prescribed mean curvature. Math. Z. 139(1974), 173-198. 
5. D. Gilbarg and N. S. Trudinger, Elliptic partial differential equations of second order. Second edition. Grundlehren der Mathematischen Wissenschaften [Fundamental Principles of Mathematical Sciences], 224. Springer-Verlag, Berlin, 1983.

6. E. Giusti, Generalized solutions for the mean curvature equation. Pacific J. Math. 88(1980), No. 2, 297-321.

7. F. LiAnG, Harnack's inequalities for solutions to the mean curvature equation and to the capillarity problem. Rend. Sem. Mat. Univ. Padova 110 (2003), 57-96.

8. F. LiANG, Global boundedness, interior gradient estimates, and boundary regularity for the mean curvature equation with boundary conditions. Int. J. Math. Math. Sci. 2004, No. 17-20, 913-948.

9. V. G. Ma'zya, Sobolev spaces. (Translated from the Russian) Springer Series in Soviet Mathematics. Springer-Verlag, Berlin, 1985.

10. L. Simon, Global estimates of Hlder continuity for a class of divergence-form elliptic equations. Arch. Rational Mech. Anal. 56(1974), 253-272.

11. L. Simon, Boundary regularity for solutions of the non-parametric least area problem. Ann. of Math. (2) 103(1976), No. 3, 429-455.

12. L. Simon and J. SPRUCK, Existence and regularity of a capillary surface with prescribed contact angle. Arch. Rational Mech. Anal. 61(1976), No. 1, 19-34.

13. G. Stampacchia, Èquations elliptiques du second ordre à coefficients discontinus. Séminaire de Mathématiques Supérieures, No. 16 (Été, 1965) Les Presses de l'Université de Montréal, Montreal, Que, 1966.

(Received 22.03.2004)

Author's address:

Institute of mathematics

Academia Sinica

Nankang, Taipei, Taiwan 11529

E-mail: liang@math.sinica.edu.tw 